\title{
FLAT FLUIDICS: PROGRAMMABLE ON-CHIP NETWORKS FOR BIOLOGICAL AND CHEMICAL APPLICATIONS
}

\author{
A. Wixforth ${ }^{1}$, Ch. Strobl ${ }^{1}$, Ch. Gauer ${ }^{2}$, A. Toegl ${ }^{2}$, J. Scriba ${ }^{2}$ and Z.V.Guttenberg ${ }^{2}$ \\ ${ }^{1}$ University of Augsburg, Experimentalphysik I, D-86159 Augsburg, Germany \\ ${ }^{2}$ Advalytix AG, Eugen-Sänger-Ring 4, D- 85649 Brunnthal, Germany
}

\begin{abstract}
Surface acoustic waves are used to actuate and process smallest amounts of fluids on the planar surface of a piezoelectric chip. Chemical modification of the chip surface is employed to create virtual wells and tubes to confine the liquids. Lithographically modulated wetting properties of the surface define a fluidic network, in analogy to the wiring of an electronic circuit. Acoustic radiation pressure exerted by the surface wave leads to internal streaming in the fluid and eventually to an actuation of small droplets along predetermined trajectories. This way, in analogy to microelectronic circuitry, programmable biochips for a variety of assays on a chip have been realized.
\end{abstract}

\section{Introduction}

The knowledge of the biochemical interior of living cells steadily increases and researchers dig deeper and deeper into the biomolecular world. Now the human genome is sequenced, scientists hope to find novel drug targets once the code is cracked. Analytical techniques like gene expression analysis or cell assays have become standard tools, used in large scale screening for new drugs. The very same technologies are the driving force behind a miniaturization of the laboratories, as parallel screening requires smallest amounts of sample for the single experiments. The resources of many of the precious ingredients are either very limited or prohibitively expensive.

In this article, we wish to report a novel way of tackling the need to scale chemical and biological laboratories down to the size of a thumbnail. We describe a technique which uses virtual beakers and channels to confine smallest amounts of liquids to the plane surface of a microchip, and tiny earthquakes (Surface Acoustic Waves, SAW) on this very chip to act as electrically adressable and programmable nanopumps. The combination of both can be viewed as a step towards the realization of a programmable microfluidic processor.

\section{Experimental Details}

\subsection{Surface Acoustic Waves}

Surface acoustic waves have been first described in combination with earthquakes [1]. Meanwhile, reduced to the significantly smaller nano scale, they found their way into much friendlier fields: SAW devices are widely used for RF signal processing and filter applications and became a huge industry in mobile communication [2]. SAW devices have been around for years in communication circuitry - every cell phone has filters using the effect. An electrical 
signal fed into so-called transducers on the surface of a piezoelectric chip is converted into a deformation of the crystal underneath. Given the right frequency of the signal, a mechanical wave is launched across the chip. In Fig. 1, we sketch a snapshot of a SAW propagating on a solid. The SAW in this figure is characterized by subsequent regions of compressed and expanded material as indicated in gray scale.

In the recent past, SAW have also been used to act in a completely different way than for filtering and signal processing just by converting electrical signals into mechanical vibrations and vice versa. Excited on piezoelectric substrates, they are accompanied by large electric fields. Those electric fields are travelling at the speed of sound of the substrate (approx. $3000 \mathrm{~m} / \mathrm{sec}$ ) having the same spatial and temporal periodicity as their mechanical companions. Charges at or close to the surface are coupling to these electric fields, and currents are induced withing a conducting layer. Nearly twenty years ago we thus introduced SAW to study the dynamic conductivity $\sigma(\omega, \mathrm{k})$ of low-dimensional electron systems in high magnetic fields and at low temperatures.

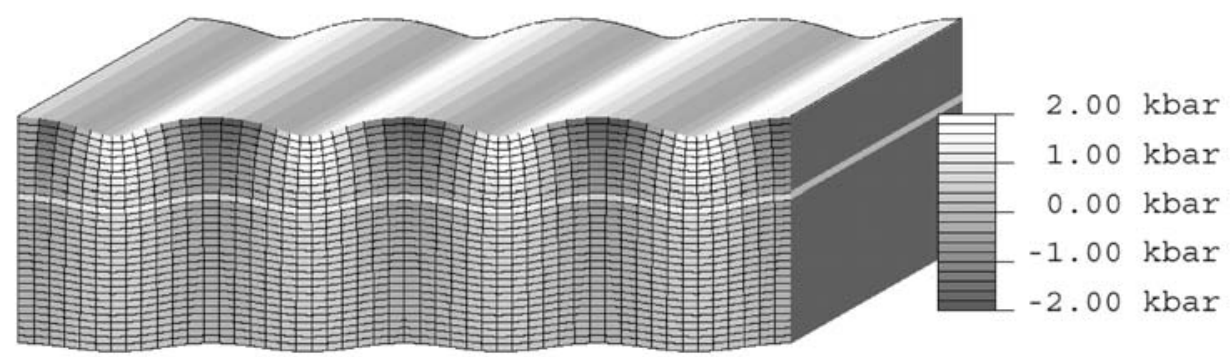

Fig. 1: Sketch of a Surface Acoustic Wave propagating on a piezoelectric substrate. Typical wavelengths are in the micrometer range, typical amplitudes less than a nanometer.

It turns out that the interaction between a SAW and the mobile charges in a semiconductor is strongest for very low sheet conductivities as they are observed, e.g., in the regime of the quantum Hall effect [3]. However, SAW can not only be used to probe the properties of quantum systems, but also to deliberately alter some of them as SAW represent a spatially modulated strain and stress field accompanied by strong electric fields in a solid, and propagating at the speed of sound. Such an interaction between SAW and the optical properties of a semiconductor quantum well led us to the discovery that photogenerated electron hole pairs in a semiconductor quantum well can be spatially separated under the influence of a SAW mediated electric field. This, in turn has an enormous impact on the photoluminescence (PL) of the semiconductor. We were able to show that the PL not only is quenched under the influence of a SAW, but also can be reestablished at a remote location on the sample and after a certain delay time [4]. Further studies include the acoustic charge transport and the creation of dynamically induced electron wires, as well as the study of nonlinear acoustic interaction with low dimensional electron systems in semiconductors [5].

However, the piezoelectric effect is usually only a small contribution to the elastic properties of a solid: Most of the energy propagating in a SAW (usually more than 95\%) is of mechanical nature. Hence, not only electrical interactions as described above, but also mechanical interactions are a possible scope for experimental investigations. Having wavelengths of a few microns and amplitudes of about only a nanometer, however, the forces and electric 
fields within the nanoquake are sufficient to have a macroscopic effect. Any piece of matter at the surface along the way of a SAW experiences their vibrating force: Viscous materials like liquids absorb a lot of their energy. It turns out that the interaction between a SAW and a liquid on top of the substrate surface induces an internal streaming, and, as we will point out below, at large SAW amplitudes this can even lead to a movement of the liquid as a whole.

The origin of such an acoustically induced internal streaming is depicted schematically in Fig. 2: A SAW is propagating from left to right along the $\mathrm{x}$-axis. At $\mathrm{x}=0$, it reaches the boundary of a liquid at the surface of the substrate. A SAW with a non vanishing amplitude in the zdirection, i.e. normal to the surface of the substrate, is then strongly absorbed by the fluid, as indicated by the decaying amplitude for positive $\mathrm{x}$ values.

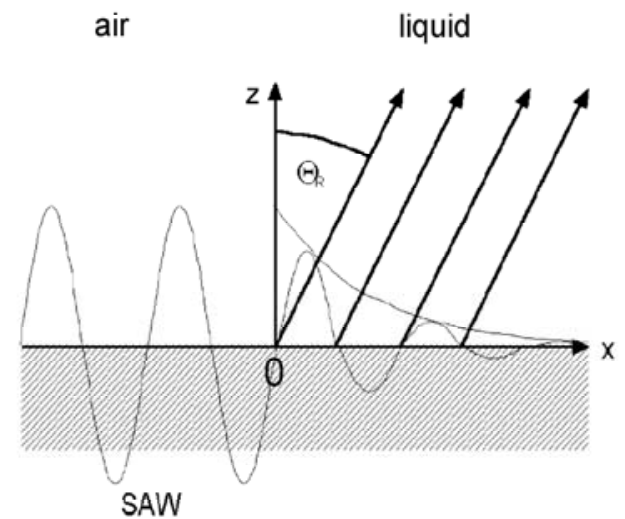

Fig. 2: Schematic illustration of the interaction between a SAW and a liquid at the surface of the SAW substrate. The SAW is propagating from left to right, and at $\mathrm{x}=0$ hits the liquid. A longitudinal sound wave is radiated into the fluid under a refraction angle $\Theta_{R}$.

Moreover, it creates a small but finite pressure difference $2 \Delta p$ in the fluid between the ridges and the wells of the wave, which transforms into a small but finite difference $2 \Delta \rho$ in the liquid density. Both quantities then spatially and temporally oscillate around their respective equilibrium value $\mathrm{p}_{0}$, and $\rho_{\mathrm{o}}$, respectively. The pressure difference directly above the surface of the substrate leads to the excitation of a longitudinal sound wave into the liquid. As the sound velocities for the liquid and the solid substrate are in general not equal, this wave is launched under a diffraction angle $\Theta_{\mathrm{R}}$, given by:

$$
\Theta_{R}=\arcsin \left(\frac{\mathrm{v}_{\mathrm{S}}}{\mathrm{v}_{\mathrm{f}}}\right)
$$

Here, $v_{\mathrm{s}}$ and $\mathrm{v}_{\mathrm{f}}$ denote the sound velocities of the substrate, and the fluid, respectively. In addition, the SAW is responsible for the build-up of an acoustic radiation pressure [6]

$$
P_{S}=\rho_{0} v_{S}^{2}\left(\frac{\Delta \rho}{\rho_{0}}\right)^{2}
$$

in the direction of the sound propagation in the fluid. This leads to an internal streaming in a closed volume like a droplet, as the boundary of the droplet reflects the actuated fluid back to the source.

Such internal SAW driven streaming in a small droplet can be nicely visualized by dissolving a dried fluerescent dye under the influence of a SAW. In Fig. 3, we depict two 
snapshots of such a fluorescence experiment, taken approximately one second apart from each other.
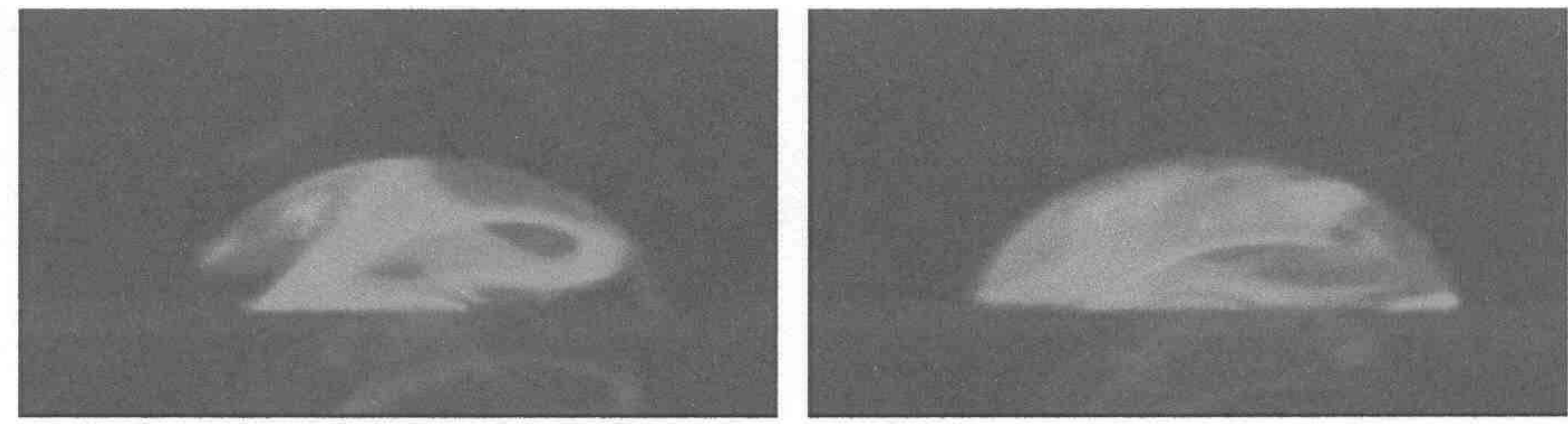

Fig. 3: SAW induced internal streaming in a small water droplet (side view, approx. $50 \mathrm{nl}$ ). A dried fluorescent dye on the surface of the chip is dissolved by SAW agitation, and rapidly fills the whole droplet volume.

For larger SAW amplitudes, the acoustic radiation pressure even deforms the droplet surface at the opposite side of the sound entrance. This can be seen in Fig. 4, where we show a droplet under the influence of a quite intense SAW, impinging from the left. As can be seen from the figure, the acoustic radiation pressure in this case is so high that it strongly deforms the droplet. At the same time, the wetting angles to the left and the right of the droplet (i.e. ,luff and lee" of the SAW) are modified with respect to the equilibrium values. This acoustically driven deformation of the droplet is the main reason for the droplet actuation in our case.
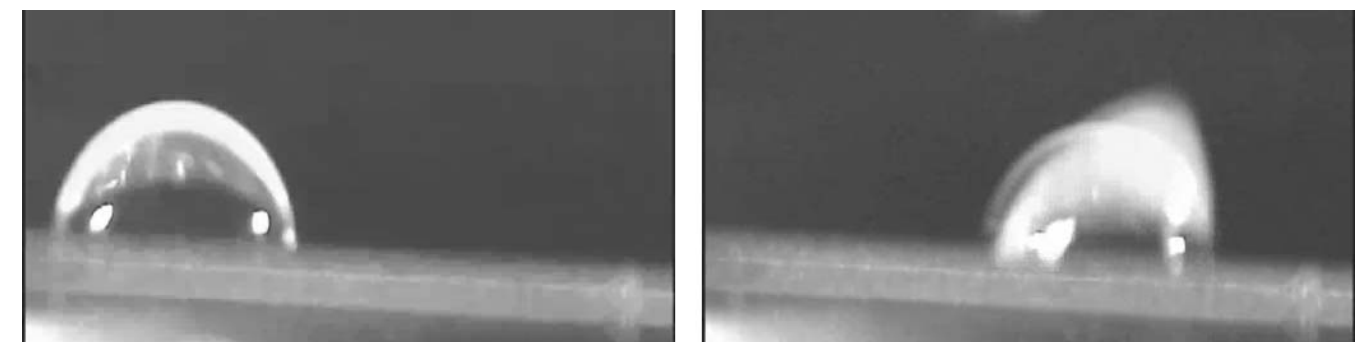

Fig. 4: Side view of a small droplet (ca. $50 \mathrm{nl}$ ) on the surface of a piezoelectric substrate. Left: Droplet in rest, note the wetting angle of about $90^{\circ}$, which has been obtained by a hydrophobic treatment of the surface. Right: droplet being ,hit“ by a SAW impinging from the left. The acoustic radiation strongly deforms the droplet shape. This leads to a momentary asymmetry of the wetting angles of the droplet.

\subsection{Virtual Containers and Tracks for Liquids on a Chip}

The next step towards an application of SAW in microfluidics in general is to create,flatland" [7] analogs to channels, tubes, reservoirs, mixing chambers and similar building blocks usually employed to guide, contain or process liquids in a fluidic network.

By a chemical modification of parts of the chip surface (e.g. silanization employing an OTS based surface chemistry) we are able to create patterns of preferred wetability (hydrophilic regions), being separated by regions of surface chemistry, where fluids are repelled (hydrophobic 
areas). Employing lithographic techniques borrowed from semiconductor microelectronics, we thus can create completely flat, two-dimensional fluidic networks, where liquids are confined to virtual tracks, reservoirs and reaction chambers by surface tension alone. There is a whole variety of methods to modulate the wetting properties of the surface of a chip: the most common technique is to silanize the surface on specific areas, whereas other regions of the surface are protected by a laterally structured photo resist, that has been exposed using a mask an UV radiation and subsequently developed leaving the exposed areas etched through to the chip surface.

In Fig. 5, we depict some of such self assembled virtual potential wells for fluids on a surface. Photolithographic techniques have been employed to create ,containers' for smallest amounts of liquid, having predetermined shapes like a hexagon (left) or a ,tube "with a narrowing (acting as a pressure driven valve, by the way). Given this surface functionalization, either closed fluid volumes or single droplets may be acoustically guided along predetermined pathways along the surface of the chip.
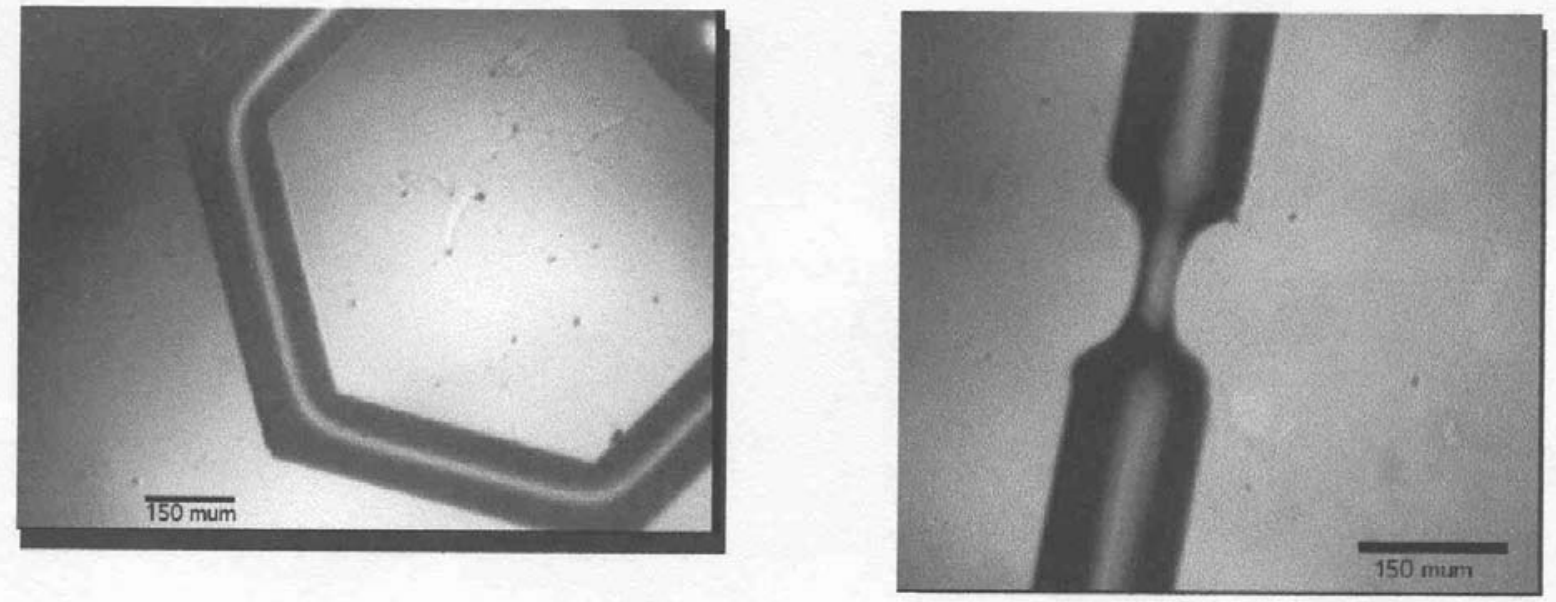

Fig. 5: Photolithografically defined surface modification to modulate its wetability. This way, , virtual' fluidic tracks are created to confine smallest amounts of liquid to predetermined geometries or to guide a SAW driven droplet along a predetermined path on the chip surface.

Given such chemically defined virtual tracks and the SAW based actuation, we thus can move several droplets of different fluids (or generally different reagents) independently into any desired direction. In Fig. 6, we give a series of snapshots of one of our fluidic chips, where three droplets are actuated using SAW agitation. Depending on the actual layout of the chip, the droplets can be split into smaller ones, they can be merged, mixed and processed in most any fashion. As the SAW nanopumps are electrically adressable, a complete sequence of different steps of a chemical ,experiment" or a biological assay can be computer controlled. Moreover, the simplicity of the fabrication process of our , programmable bioprocessors" makes them a serious candidate for truly miniaturized laboratories on a chip [8]. 


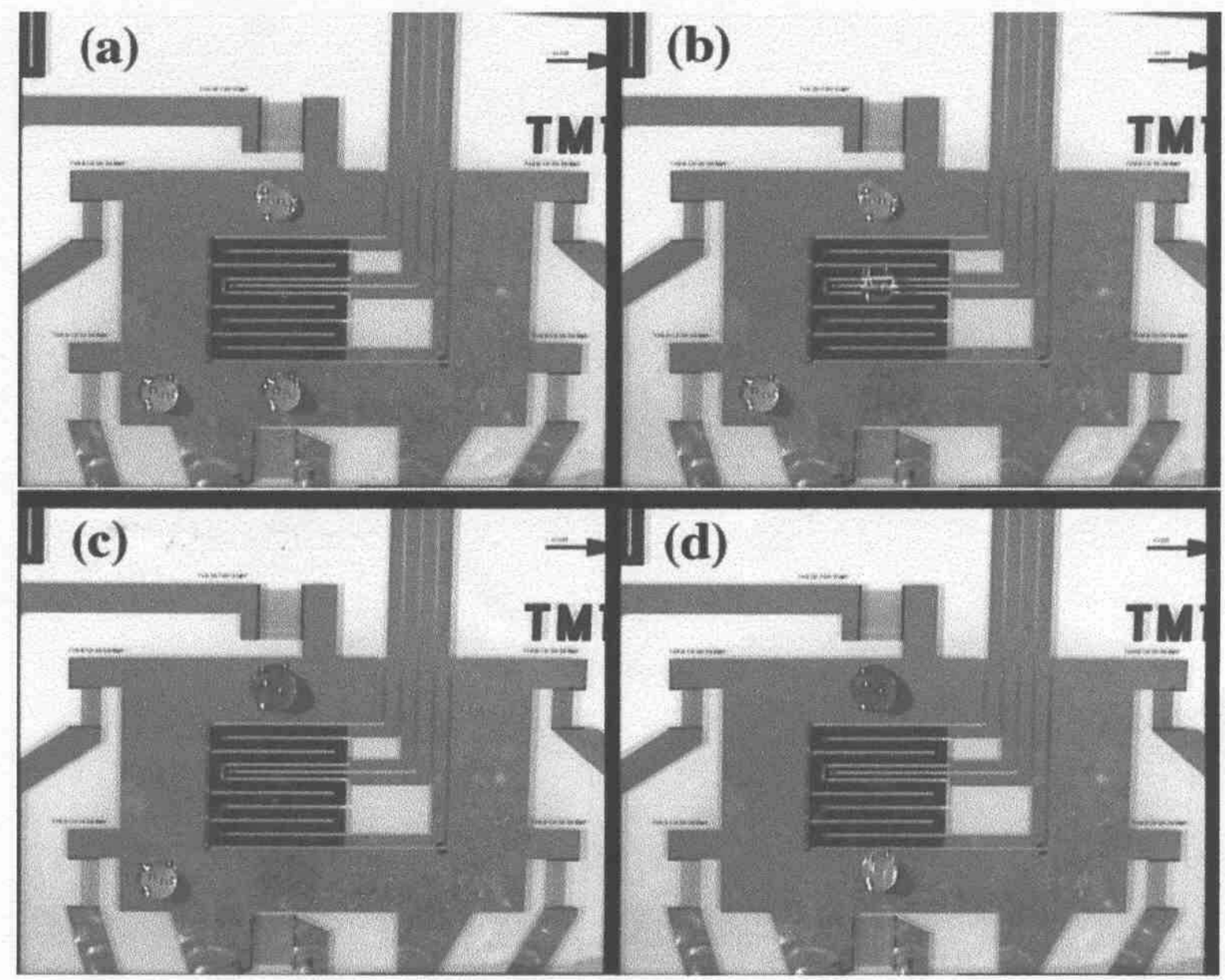

Fig. 6: A SAW driven microfluidic processor. Three droplets (approx. $100 \mathrm{nl}$ each) are moved ,remotely controlled' and independently by the nanopumps. (a) through (d) represent a series of subsequently taken snapshots to visualize the movement, and the ,nanochemical reactions' ocurring when the droplets are merged and mixed by the action of the surface wave. The chip not only contains the nanopumps and the fluidic environment but in the center additional real estate like sensors, and heaters.

It turns out, that the interaction between a SAW and a droplet on a flat surface of a chip is strongly non-linear in terms of the ratio between the SAW wavelength and the droplet diameter. In other words, for a given SAW wavelength and power, only droplets of a certain volume can be effectively actuated along a fluidic track, whereas droplets of a smaller volume remain basically unaffected by the acoustic pressure in this case. This effect can be used to create very small droplets (approx. 50 picoliters) out of a larger one (approximately 50 nanoliters) by leaving them behind on a microstructured chemically modified surface. Such an array of ultrasmall droplets can be regarded as a special form of a microarray. For special applications, also a layer-by-layer build up of complex sandwich systems might be generated employing this scheme. 


\section{Applications}

\subsection{SAW Driven Agitation of Hybridization Assays}

First applications of the SAW based fluidic actuation already exist and are presently commercialized (ArrayBooster $^{\mathrm{TM}}$, SlideBooster ${ }^{\mathrm{TM}}$, Advalytix AG, Brunnthal, Germany): the ability of SAW to efficiently stir and mix smallest amounts of fluids is employed to enhance the results of biological hybridization assays. During hybridization, immobilized DNA fragments or oligonucleotides which are spotted on a microarray, are flooded by an unknown sample fluid, containing other oligonucleotides. Once hybridization occurs, fluorescence markers at the sample molecules accumulate at a specific spot. This fluorescent signal can then be detected and act as a measure for the hybridization efficiency, in other words, the degree of matching between sample and target molecule.

Usually, such micro array hybridizations are performed in a thin capillary fluid film (thickness approx. $50 \mu \mathrm{m}$ ), spread across the area of a conventional microscope slide $(7.5 \mathrm{~cm} * 2.5 \mathrm{~cm})$. Here, the narrowness of the film again leads to a complete suppression of turbulent flow in the film, diffusion is the only driving force to move a sample molecule towards a target spot.

Table 1: Calculated diffusion times for different diffusion lengths and three different particle sizes. A DNA segment of only $100 \mathrm{~nm}$ lengths needs about 30 hours to diffuse over a distance of only 1 $\mathrm{mm}$.

\begin{tabular}{|c|c|c|c|}
\hline $\begin{array}{c}\text { Diffusion length } \\
(\mu \mathrm{m})\end{array}$ & $\begin{array}{c}\text { Potassium ion } \\
(0.2 \mathrm{~nm})\end{array}$ & $\begin{array}{c}\text { Oligonucleotide } \\
(6 \mathrm{~nm})\end{array}$ & $\begin{array}{c}\text { PCR product } \\
(100 \mathrm{~nm})\end{array}$ \\
\hline 1 & $0.2 \mathrm{~ms}$ & $6 \mathrm{~ms}$ & $100 \mathrm{~ms}$ \\
\hline 10 & $20 \mathrm{~ms}$ & $600 \mathrm{~ms}$ & $10 \mathrm{~s}$ \\
\hline 100 & $2 \mathrm{~s}$ & $60 \mathrm{~s}$ & $20 \mathrm{~min}$ \\
\hline 1000 & $200 \mathrm{~s}$ & $100 \mathrm{~min}$ & $30 \mathrm{~h}$ \\
\hline
\end{tabular}

In table 1, for instance, we have calculated the diffusion time needed to overcome a certain distance in such a thin liquid film. To cover a distance of only $1 \mathrm{~mm}$, a $100 \mathrm{~nm}$ long DNA fragment needs already approximately 30 hours. This diffusion limit together with the then unavoidable depletion effects leads to a very slow hybridization process for microarrays. Obviously, mixing and stirring the fluid in the narrow slit would improve this process, as the diffusion limit can be overcome in this case. Although SAW mediated agitation in a thin liquid film is rather slow as compared to a free fluid surface, the diffusion limit can be definitely overcome as we show in Fig. 7. Here, we depict the result of the fluorescence analysis of a typical microarray assay for the diffusion only, and the mixed case. Not only do we observe a quite dramatic increase of the signal intensity in the latter case, also the homogeneity of the single spots on the microarray is much better for the agitated sample [8]. 


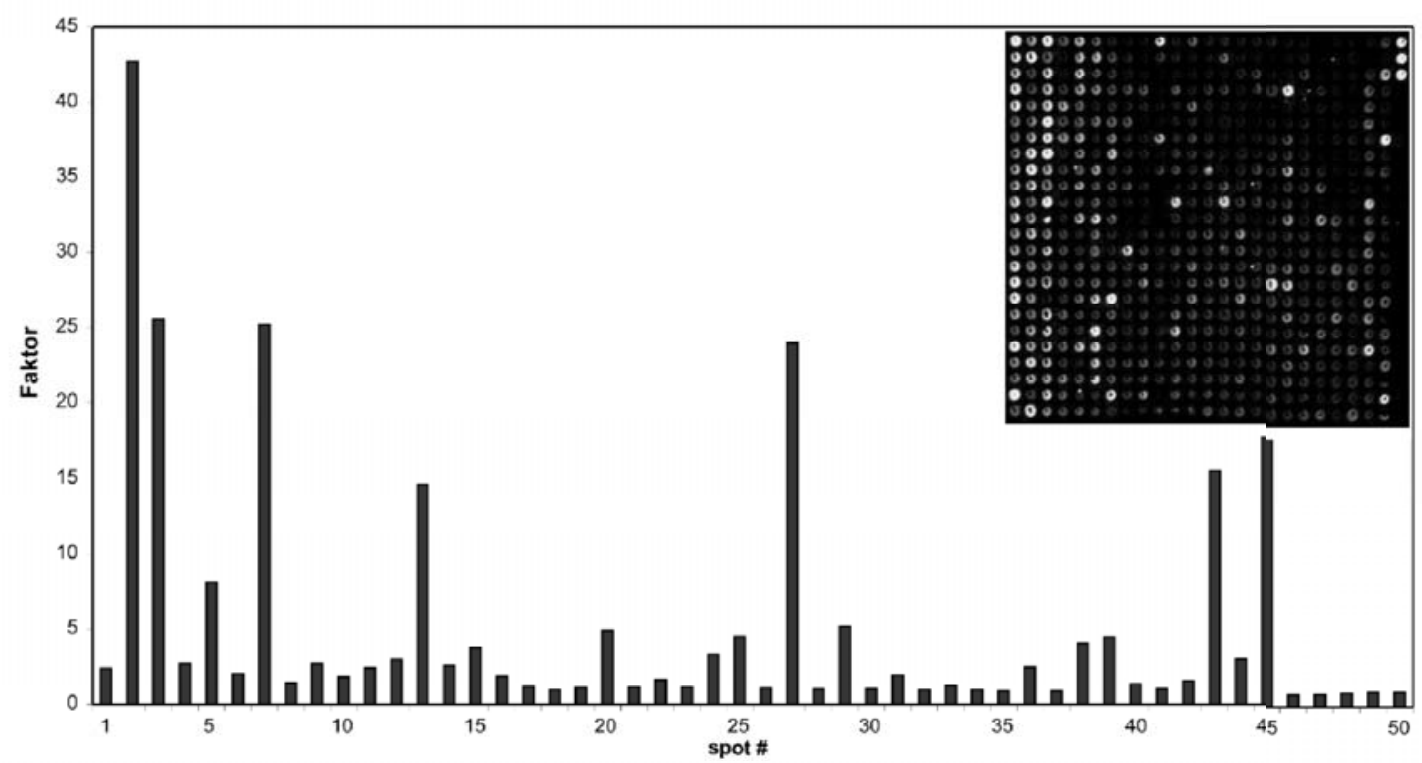

Fig. 7: Result of a flourescence labelled micro array experiment (rat chip with 50 oligos, three subarrays, two replicas each, overnight hybridization at $42^{\circ} \mathrm{C}$ ). We show the intensity ehancement for different spots on the microarray for the agitated sample fluid as compared to the diffusion limited case. The inset shows a typical microarray.

\subsubsection{PCR on a Chip}

The ability of our novel, acoustically driven, programmable bioprocessors to actuate small liquid droplets, to stir them during hybridization, etc. led us to the attempt to perform a complete polymerase chain reaction (PCR) on a chip.

For this purpose, in co-operation with the Institute for Physical High Technology (IPHT) in Jena, Germany, we have integrated the fluid handling techniques as described above together with a micro heater and a thermometer on the planar surface of the chip. Heating and cooling rates of the order of $5 \mathrm{~K} / \mathrm{sec}$ have been achieved on this chip.

As a proof of concept, a standard PCR protocol (genomic DNA with STS primer, main product 150 base pairs) has been scaled down to a droplet size of less than 1 microliter. A PCR mix containing polymerase, primer, d-NTP, template DNA, and reaction buffer formed the sample droplet. To prevent this small droplet from evaporation, it has been covered by a small amount of mineral oil. Thus, the sample droplet and the oil cover formed a virtual micro reactor which could be actuated as a whole across the chip surface. The technological challenge behind this process was to simultaneously define hydrophobic and lipophobic areas and tracks on the chip surface. In the present case (see upper left inset of Fig. 8), we achieved a wetting angle of about $90^{\circ}$ for both fluids.

Both on-chip microarray detection as well as a subsequent gel electrophoretic test showed that PCR on a chip is feasibe and that after 30 cycles even only three start copies in the droplet could be successfully amplified. As a very attractive addition to this on-chip PCR we were also able to show that a multi-spot PCR is also possible. For this purpose, up to four different sample droplets have been covered by oil, simultaneously (see Fig. 8, center). 


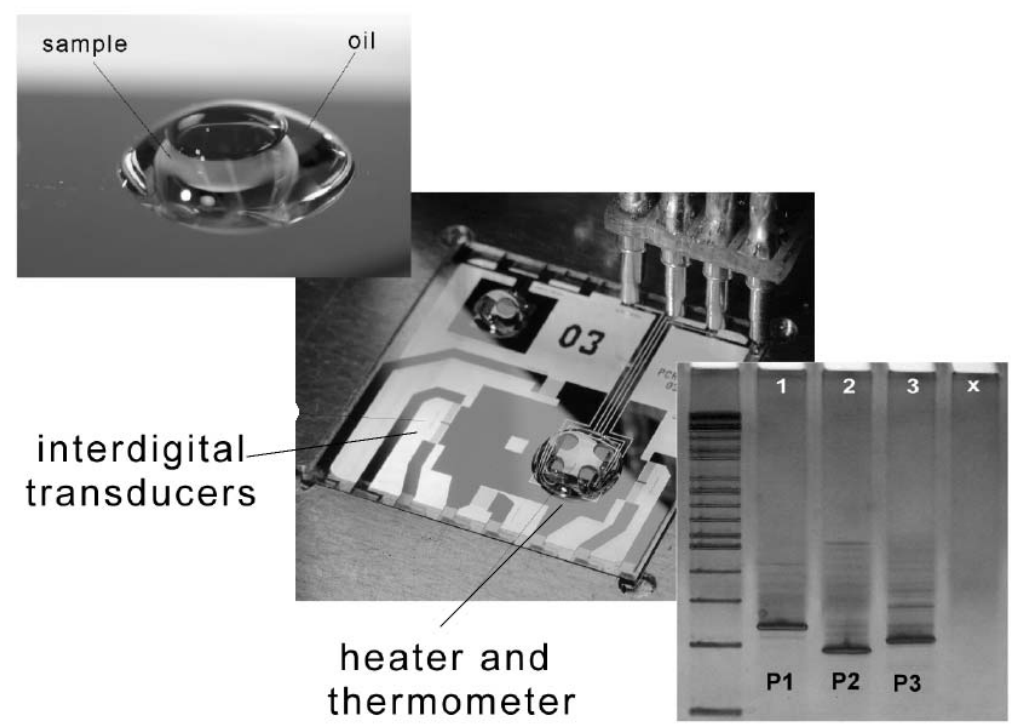

Fig. 8: Chip based polymerase chain reaction (PCR) as a proof of concept for a programmable bioprocessor. On the chip (center), interdigital transducers for the acoustic manipultion of the small droplets as well as a heater and a thermometer have been integrated simultaneously. Four different PCR protocols have been performed at the same time, employing a „multi-spot" technique as described in the text. To prevent the small sample droplets (less than a microliter) from evaporation during the PCR cycles, all four droplets have been covered simultaneously by an oil drop (see also upper inset). A subsequent gel electrophoresis shows, that no cross contamination between the droplets (samples P1 through P3 and a control droplet, lane X) occurs.

The PCR, too, was performed simultaneously for the four droplets. Subsequent analysis showed no cross contamination between the single droplets (see Fig. 8, lower right inset)!

A very important feature of the on-chip polymerase chain reaction is that we are able to monitor the progress of the amplification in situ - again mediated by the SAW driven agitation which favours the dynamic processes involved.

The on-chip PCR is still under evaluation but we anticipate a detailed description of the whole process in the near future.

\section{Summary}

In summary, we have described a novel and unconventional method for microfluidic fluid handling at very small sample volumes. Surface acoustic waves on a piezoelectric substrate strongly interact with a liquid at the surface of this substrate which leads to the build up of an acoustic radiation pressure in the fluid. This pressure is basically the origin for the SAW mediated internal streaming in the fluid as well as for the actuation of small droplets as a whole. Laterally patterned surface modification in addition enables the creation of fluidic tracks, containers, or functional elements for a planar microfluidic network. Programmable actuation of different small droplets together with a wealth of possible fluidic functional blocks denotes a step towards the realization of a programmable fluidic microprocessor. 
This work was sponsored in part by the German Bundesministerium für Bildung und Forschung, BMBF, and in part by the Bayerische Forschungsstiftung under the program „FORNANO“.

\section{References}

[1] Lord Rayleigh, Proc. London Math Soc. 17, 4 (1885)

[2] B.A. Auld, ,Acoustic Fields and Waves in Solids“, John Wiley \& Sons, New York, 1973

[3] A. Wixforth, J.P. Kotthaus and G. Weimann, Phys. Rev. Lett. 56, 2104 (1986)

[4] C. Rocke, S. Zimmermann, A. Wixforth, J.P. Kotthaus, G. Böhm and G. Weimann, Phys. Rev. Lett. 78, 4099 (1997)

[5] M. Rotter, A.V. Kalameisev, A.O. Govorov, W. Ruile and A. Wixforth, Phys. Rev. Lett. 82, 2171 (1999)

[6] M. Rotter, A. Wixforth, A.O. Govorov, W. Ruile, D. Bernklau and H. Riechert, Appl. Phys. Lett. 75, 965 (1999)

[7] Lord Rayleigh, Phil. Mag. 10 Series 6, 364-374 (1905).

[8] „Flatland: A romance of many dimensions“ by Edwin A. Abbott (1894)

[9] A. Wixforth, J. Scriba and C. Gauer, mstNews 5/2002, 42 (2002).

[10] A. Toegl, R. Kirchner, C. Gauer and A. Wixforth, Journal of Biomolecular Techniques, 14:197-204 (2003) 